\title{
Cosmic test backs 'quantum spookiness'
}

\author{
Physicists harness starlight to support the case for entanglement.
}

\section{Elizabeth Gibney}

02 February 2017

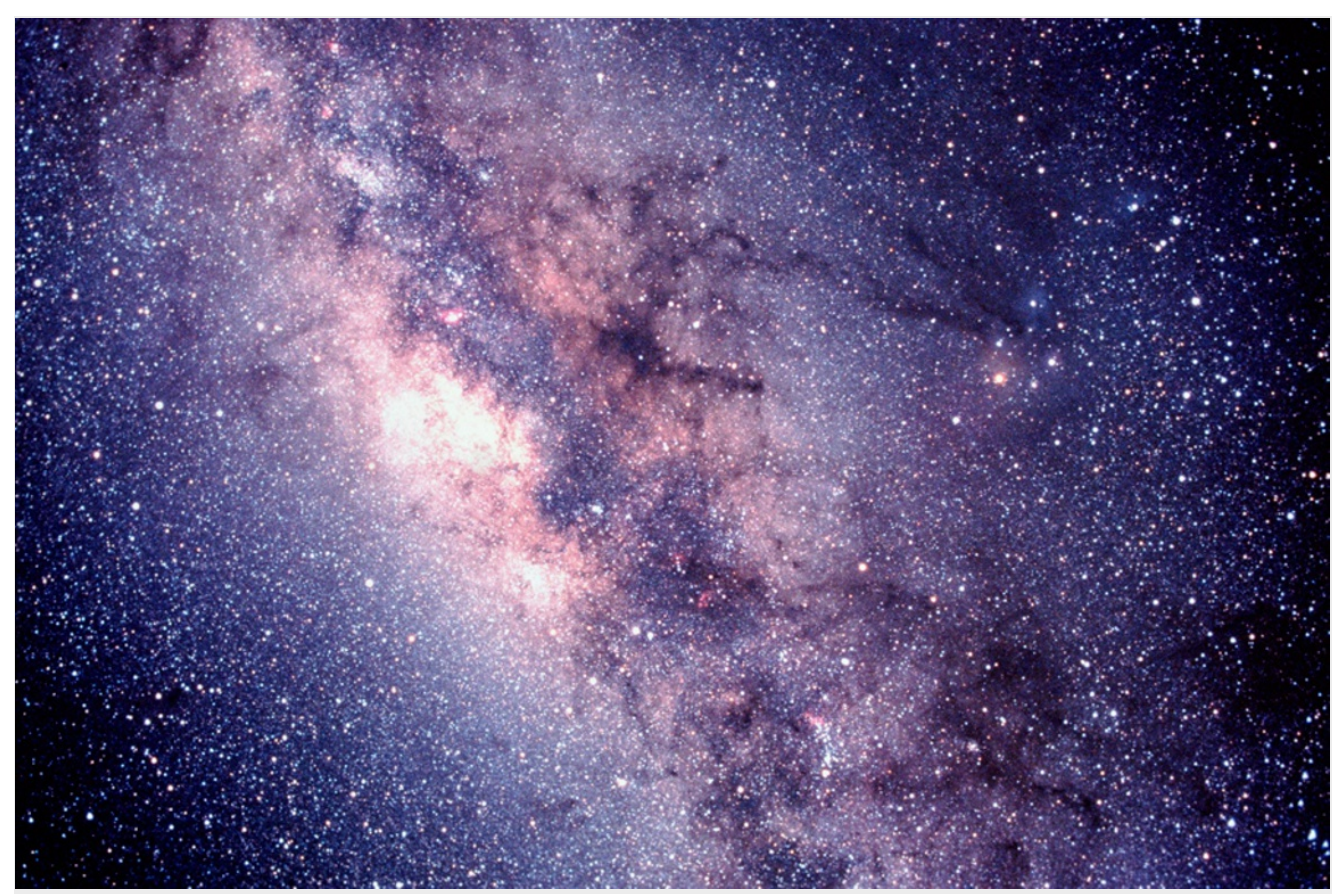

Dr Fred Espenak/Science Photo Library

The light from distant stars is used to fix settings in a new version of the iconic Bell test.

A version of an iconic experiment to confirm quantum theory has for the first time used the light of distant stars to bolster the case for a phenomenon that Albert Einstein referred to as "spooky action at a distance".

Einstein disliked the notion that objects can share a mysterious connection across any distance of space, and scientists have spent the past 50 years trying to make sure that their results showing this quantum effect could not have been caused by more intuitive explanations.

Quantum physics suggests that two so-called entangled particles can maintain a special connection — even at a large distance such that if one is measured, that instantly tells an experimenter what measuring the other particle will show. This happens despite the fact neither particle has definite properties until it is measured. That unsettled some physicists, including Einstein, who favoured an alternative explanation: that quantum theory is incomplete, and that the outcomes instead depend on some predetermined, but hidden, variables.

The latest effort to explore the phenomenon, to be published ${ }^{1}$ in Physical Review Letters on 7 February, uses light emitted by stars around 600 years ago to select which measurements to make in a quantum experiment known as a Bell test. In doing so, they narrow down the point in history when, if they exist, hidden variables could have influenced the experiment.

"It's a beautiful experiment," says Krister Shalm, a quantum physicist at the US National Institute of Standards and Technology (NIST) in Gaithersburg, Maryland. Although few expected it to disprove quantum mechanics, such experiments "keep pushing alternative theories to be more and more contrived and ridiculous", he says. Similar techniques could, in the future, help to protect against hackers who try to crack quantum-cryptography systems, he adds.

\section{Closing loopholes}

Physicists at the University of Vienna, along with colleagues in China, Germany and the United States, developed a new version of the Bell test - a protocol devised by the physicist John Bell in the 1960s to distinguish between two possible explanations for the 
seemingly strange behaviour of the quantum world.

The test involves performing independent measurements on separated pairs of entangled quantum particles. Bell showed that, statistically, correlations between the results, once above a certain threshold limit, could not be explained by particles having hidden properties. Instead the coordinated outcomes seem to be the result of measurements on one particle mysteriously fixing the properties of the other.

Although Bell tests have supported quantum theory many times, they include assumptions that leave wiggle room for non-quantum explanations, and physicists have been trying to close these 'loopholes' ever since.

"We outsource the choice to the Universe itse If."

In 2015, they sealed a major victory when three separate teams, including Shalm's, succeeded in simultaneously closing two major possible loopholes, by showing that entanglement could not be an illusion created by any speed-of-light communication between particles, or an artefact of only detecting certain photons $2,3,4$

\section{Freedom of choice}

But they left open another loophole — one that is more subtle, and impossible to fully close, says Andrew Friedman, an astronomer at the Massachusetts Institute of Technology in Cambridge, and a co-author on the latest paper. Bell tests also assume that experimenters have free choice over which measurements they perform on each of the pair of photons. But some unknown effect could be influencing both the particles and what tests are performed (either by affecting choice of measurement directly, or more plausibly, by restricting the options that are available), to produce correlations that give the illusion of entanglement.

To narrow this freedom-of-choice loophole, researchers have previously put 144 kilometres between the source of entangled particles and the random-number generator that they use to pick experimental settings ${ }^{5}$. The distance between them means that if any unknown process influenced both set-ups, it would have to have done so at a point in time before the experiment. But this only rules out any influences in the microseconds before: the latest paper sought to push this time back dramatically, by using light from two distant stars to determine the experimental settings for each photon. "We outsource the choice to the Universe itself," says Friedman.

The team, led by physicist Anton Zeilinger at the University of Vienna, picked which properties of the entangled photons to observe depending on whether its two telescopes detected incoming light as blue or red. The colour is decided when the light is emitted, and does not change during travel. This means that if some unknown effect, rather than quantum entanglement, explains the correlation, it would have to have been set in motion at least around 600 years ago, because the closest star is 575 light-years (176 parsecs) away, says Friedman, who hopes to eventually push back this limit to billions of years ago by doing the experiment with light from more distant quasars. Their results found a level of correlation that supports 'action at a distance'1.

\section{Protection against hackers}

Technically, the experiment is impressive, say Ronald Hanson, a quantum physicist at the Delft University of Technology in the Netherlands. But, unlike the loopholes closed in 2015, this one can never be fully closed; confining it to further in the past is only possible by making new assumptions - in this case, for example, by assuming that no one messed with the photons immediately before they hit the telescopes, he says.

Others argue that although, fundamentally, the loophole is never closable, such experiments are valuable because new theories necessarily become more improbable and contrived, or eventually, end up assuming that everything in the Universe was determined at the time of the Big Bang - a philosophical view that most physicists reject. Reworking experiments to reduce and make better assumptions is therefore worthwhile, says Shalm.

Such experiments also have practical value, argues Friedman, because if quantum mechanics turns out to be explained by a different underlying theory, that discovery could impact the security of technologies that rely on quantum theory, such as quantum encryption. And trying to close such loopholes is useful because minimizing the assumptions in an experiment serves to also beef up protection against hackers who might otherwise exploit them, says Shalm, whose team at the NIST is exploring whether Bell tests could be used in quantum cryptography.

Harnessing cosmic phenomena is not the only way physicists are ensuring the independence of their measurement settings. In November, teams from around the world took part in the Big Bell Test, which tapped 100,000 game-playing volunteers worldwide to create random sequences of $0 \mathrm{~s}$ and $1 \mathrm{~s}$, which physicists used to fix their measurement settings. 
Preliminary analysis indicates that in this case, most — and possibly even all — of the experiments yet again supported quantum mechanics, says Morgan Mitchell at the Institute of Photonic Sciences (ICFO) in Barcelona, Spain, which coordinated the event. "Sorry, Einstein," he says.

Nature | doi:10.1038/nature.2017.21401

\section{References}

1. Handsteiner, J. et al. Preprint at https://arxiv.org/abs/1611.06985 (2016).

2. Shalm, L. K. et al. Phys. Rev. Lett. 115, 250402 (2015).

3. Hensen, B. et al. Nature 526, 682-686 (2015).

4. Giustina, M. et al. Phys. Rev. Lett. 115, 250401 (2015).

5. Scheidl, T. et al Proc. Natl Acad. Sci. 107 19708-19713 (2010) 